Communications in Physics, Vol. 27, No. 1 (2017), pp. 71-82

DOI:10.15625/0868-3166/27/1/9221

\title{
THERMODYNAMIC HADRON-QUARK PHASE TRANSITION OF CHIRAL NUCLEAR MATTER TO QUARK-GLUON PLASMA
}

\author{
NGUYEN TUAN ANH ${ }^{\dagger}$ \\ Faculty of Energy Technology. Electric Power University, \\ 235 Hoang Quoc Viet, Hanoi, Vietnam \\ ${ }^{\dagger}$ E-mail: dr.tanh@gmail.com
}

Received 14 February 2017

Accepted for publication 01 March 2017

\begin{abstract}
After receiving very interesting results from investigations of chiral nuclear matter based on the extended Nambu-Jona-Lasinio model (ENJL) included the scalar-vector eight-point interaction, a fundamental question of nuclear physics is what happens to chiral nuclear matter as it is compressed or heated. At very high density and temperature, quarks and gluons come into play and a transition is expected to happen from a phase of nuclear matter consisting of confined hadrons and mesons to a state of 'liberated' quarks and gluons. In this paper, we investigate the hadron-quark $(H Q)$ phase transition occurs beyond the chiral phase transition in the nuclear matter. The results show that there exits a quarkyonic-like phase, appeared just before deconfinement, when the chiral symmetry is restored but the elementary excitation modes are still nucleonic.
\end{abstract}

Keywords: nuclear matter, equations of state of nuclear matter, chiral symmetries, bag model, quark-gluon plasma, quark deconfinement, equilibrium properties near critical points, phase transitions and critical phenomena.

Classification numbers: 21.65.-f, 21.65.Mn, 11.30.Rd, 12.39.Ba, 12.38.Mh, 25.75.Nq, 64.60.F-, 68.35.Rh.

\section{INTRODUCTION}

Recently, the mechanism of the hadron-quark (HQ) deconfinement is one of hot topics in modern physics. The confinement mechanism is a intrinsic property of quantum chromodynamics (QCD). It is an effect of asymptotically free theory [1], i.e. as the exchanged momentum increases or as the mutual distance decreases, interactions between quarks and gluons become weaker. It means, at very high density and/ or temperatures [2] the interactions which confine quarks and gluons inside hadrons should become sufficiently weak to release them. The phase where quarks and gluons are deconfined at very highdensity and/ or temperatures is termed the quark-gluon plasma (C)2017 Vietnam Academy of Science and Technology 
(QGP). The existence of such a phase of strongly interacting matter at zero net-baryon density and temperatures larger than $\sim 170 \mathrm{MeV}$ has been established by lattice QCD calculations. There have been proposed and discussed various types of scenario concerned with the hadron-quark deconfinement transitions at high-density and high-temperature regions, but it is still unclear, even, whether the phase transition is the first order or the cross over [3]. Unique assumption in this paper is that the phase transition is of the first order as suggested by many model studies [4] and ours [15]. One of the direct consequences of this assumption is the emergence of the HQ mixed phase during the phase transition.

The transition between confinement and deconfinement is of the phase transition in chiral symmetry between hadronic matter and quark-gluon phase. Theoretical studies of HQ phase transition and/or the phase diagram for HQ many-body systems at finite density and temperature are the most recent interests. In extremely dense and/or hot environment for HQ systems [3] there may exist various possible phases with rich symmetry breaking pattern. The first principle lattice calculations have examined theoretically the extremely high density and/or temperature system which is reproduced experimentally by the relativistic heavy ion collisions (RHIC). However, due to the so-called sign problem in the finite density system the lattice QCD simulation is not straightforwardly feasible. Thus, to deal with finite density system an effective model based on QCD can be an useful tool. Hence, the chiral phase transition has been investigated by using the various effective models at finite density and temperature. However, due to the quark confinement on the hadron phase it is still difficult to derive the definite results on the HQ phase transition.

For the symmetric nuclear matter, it is necessary to describe the properties of nuclear saturation and chiral symmetry restoration. The saturation property of nuclear matter is described successfully by the Walecka's model [5]. The underlying microscopic mechanism for saturation is a balance between repulsive and attractive forces among nucleons at this particular value of the baryon density. Although this model has given many successful results for nuclear matter and finite nuclei, at first stage this model has no chiral symmetry - a symmetry plays an important role in QCD. On the other hand, one of the successful effective models of QCD related to the chiral symmetry and the dynamical chiral symmetry breaking is the Nambu-Jona-Lasinio (NJL) model [6]. NJL model gives many important results for hadronic world [7] and the dense quark matter $[8,9]$. Hence, NJL model is used to investigate the stability of nuclear matter [10], and beyond the mean-field theory $[11,12]$. Unfortunately, the nuclear saturation property cannot be reproduced if starting from the original NJL model with chiral symmetry and the nucleon is regarded as a fundamental fermion, not composite one. Fortunately, the nuclear saturation property is well reproduced if the scalar-vector and isoscalar-vector eight-point interactions are introduced holding the chiral symmetry in the NJL model, in which the nucleon is still treated as a fundamental fermion [13]. Recently, we reconsidered phase structures of chiral nuclear matter at finite density and temperature using an extended version of the NJL model with a scalar-vector interaction $[14,15]$. The observed saturation properties of nuclear matter at $\rho_{B}=\rho_{0}$ is reproduced well by this ENJL version. It reveals a first-order liquid-gas phase transition occurring at subsaturated densities as presented in any realistic model of nuclear matter; Moreover, the model considered by [15] predicts a restoration of chiral symmetry at high baryon densities, $\rho_{B} \gtrsim 2.2 \rho_{0}$ for $T \lesssim 171$ $\mathrm{MeV}$, and at high temperatures $T \gtrsim 171 \mathrm{MeV}$ for $\rho_{B} \lesssim 2.2 \rho_{0}$.

For the quark-gluon matter, we use the effective models of QCD such as the NJL model or the MIT bag model for quark matter have been actively done instead. We, hereafter, use the MIT 
bag model for simplicity. The quark-gluon matter undergoes a phase transition to the so called quark-gluon plasma phase (QGP) at high temperatures. We may learn more about their structure by studying how hadrons melt. So, hadrons have to be melted first, before filling the space with thermal quarks and gluons.

In this paper, the nuclear matter is described by the ENJL model [15], and QGP is described by the MIT bag model. We then construct at high temperatures a nuclear matter EoS from to that of Ref. [15] and put in equilibrium with the MIT bag EoS [16] for the QGP phase. It is expected that a HQ phase transition occurs at high density and temperature beyond the chiral symmetry restoration in nuclear matter.

This paper is organized as follows. We briefly recapitulate the ENJL model for chiral nuclear matter at finite density and temperature following Ref. [15] in the next section,. In Sec. III, the hadron-quark phase transition at high density and temperature is described basing on this model. A concluding remarks is given in the last section.

\section{THE CHIRAL NUCLEAR MATTER}

We use a modification version of the original $\sigma-\omega$ model [5] for hadronic matter, as presented in Ref. [15]. In this model, the EoS, i.e., the pressure $P$ as a function of baryochemical potential $\mu$ and temperature $T$, can be derived from the following Lagrangian investigated in the meanfield (or one-loop, or Hartree) approximation

$$
\begin{aligned}
L= & \bar{\psi}\left(i \hat{\partial}+\mu \gamma_{0}\right) \psi+\frac{G_{s}}{2}\left[(\bar{\psi} \psi)^{2}+\left(\bar{\psi} i \gamma_{5} \vec{\tau} \psi\right)^{2}\right]-\frac{G_{v}}{2}\left[\left(\bar{\psi} \gamma^{\mu} \psi\right)^{2}+\left(\bar{\psi} \gamma_{5} \gamma^{\mu} \psi\right)^{2}\right] \\
& +\frac{G_{s v}}{2}\left[(\bar{\psi} \psi)^{2}+\left(\bar{\psi} i \gamma_{5} \vec{\tau} \psi\right)^{2}\right]\left[\left(\bar{\psi} \gamma^{\mu} \psi\right)^{2}+\left(\bar{\psi} \gamma_{5} \gamma^{\mu} \psi\right)^{2}\right],
\end{aligned}
$$

where $\vec{\tau}=\vec{\sigma} / 2$ with $\vec{\sigma}$ Pauli matrices, $\mu$ is the baryon chemical potential, and $G_{s}, G_{v}$ and $G_{s v}$ are coupling constants.

At nuclear scale, fermion interactions are in bound states as so-called bosonization,

$$
\sigma=\bar{\psi} \psi, \quad \vec{\pi}=\bar{\psi} i \gamma_{5} \vec{\tau} \psi, \quad \omega_{\mu}=\bar{\psi} \gamma_{\mu} \psi, \quad \phi_{\mu}=\bar{\psi} \gamma_{5} \gamma_{\mu} \psi
$$

yielding

$$
\begin{aligned}
\mathscr{L}= & \bar{\psi}\left(i \hat{\partial}+\mu \gamma_{0}\right) \psi+\left[G_{s}+G_{s v}\left(\omega^{2}+\phi^{2}\right) \bar{\psi}\left(\sigma+i \gamma_{5} \vec{\tau} \vec{\pi}\right) \psi-\left[G_{v}-G_{s v}\left(\sigma^{2}+\pi^{2}\right)\right] \bar{\psi}\left(\hat{\omega}+\gamma_{5} \hat{\phi}\right) \psi\right. \\
& -\frac{G_{s}}{2}\left(\sigma^{2}+\pi^{2}\right)+\frac{G_{v}}{2}\left(\omega^{2}+\phi^{2}\right)-3 \frac{G_{s v}}{2}\left(\sigma^{2}+\pi^{2}\right)\left(\omega^{2}+\phi^{2}\right) .
\end{aligned}
$$

In the mean-field approximation, the $\sigma, \pi, \omega$, and $\phi$ fields have the ground state expectation values

$$
\langle\sigma\rangle=u, \quad\left\langle\pi_{i}\right\rangle=0, \quad\left\langle\omega_{\mu}\right\rangle=\rho_{B} \delta_{0 \mu}, \quad\left\langle\phi_{\mu}\right\rangle=0 .
$$

Hence,

$$
\mathscr{L}_{M F T}=\bar{\psi}\left(i \hat{\partial}-m^{*}+\gamma_{0} \mu^{*}\right) \psi-U\left(\rho_{B}, u\right),
$$


where

$$
\begin{aligned}
m^{*} & =-\tilde{G}_{s} u, \quad \tilde{G}_{s}=G_{s}+G_{s v} \rho_{B}^{2}, \\
\mu^{*} & =\mu-\left[G_{v}-G_{s v}\left(u^{2}+v^{2}\right)\right] \rho_{B}, \\
U\left(\rho_{B}, u\right) & =\frac{1}{2}\left(G_{s} u^{2}-G_{v} \rho_{B}^{2}+3 G_{s v} u^{2} \rho_{B}^{2}\right) .
\end{aligned}
$$

Based on Lagrangian (4) the thermodynamic potential is derived

$$
\Omega\left(\rho_{B}, u\right)=U\left(\rho_{B}, u\right)+2 N_{f} \int \frac{d^{3} k}{(2 \pi)^{3}}\left[E_{k}+T \ln \left(n_{-} n_{+}\right)\right],
$$

where $n_{\mp}=\left[e^{E_{\mp} / T}+1\right]^{-1}, E_{\mp}=E_{k} \mp \mu^{*}, E_{k}=\sqrt{k^{2}+m^{* 2}}$, and $N_{f}=2$ for nuclear matter and $N_{f}=1$ for neutron matter.

The ground state of nuclear matter is determined by the minimum condition

$$
\frac{\partial \Omega}{\partial u}=0
$$

or

$$
u=2 N_{f} \int \frac{d^{3} k}{(2 \pi)^{3}} \frac{m^{*}}{E_{k}}\left(n_{-}+n_{+}-1\right),
$$

which is called the gap equation.

In terms of the baryon density

$$
\rho_{B}=-\frac{\partial \Omega}{\partial \mu_{B}}=2 N_{f} \int \frac{d^{3} k}{(2 \pi)^{3}}\left(n_{-}-n_{+}\right),
$$

the equations of state read

$$
\begin{aligned}
P= & -\frac{m^{* 2}}{2 \tilde{G}_{s}}-\frac{G_{v}}{2} \rho_{B}^{2}+\left(\mu-\mu^{*}\right) \rho_{B} \\
& -2 N_{f} \int \frac{d^{3} k}{(2 \pi)^{3}}\left[E_{k}+T \ln \left(n_{-} n_{+}\right)\right], \\
\mathscr{E}= & \frac{m^{* 2}}{2 \tilde{G}_{s}}+\frac{G_{v}}{2} \rho_{B}^{2}+2 N_{f} \int \frac{d^{3} k}{(2 \pi)^{3}} E_{k}\left(n_{-}+n_{+}-1\right) .
\end{aligned}
$$

Table 1. Values of parameters and physical quantities.

\begin{tabular}{ccccccc}
\hline & $G_{s}\left(\mathrm{fm}^{2}\right)$ & $G_{v} / G_{s}$ & $G_{s v} / G_{s}$ & $m_{0}(\mathrm{MeV})$ & $m^{*} / m_{N}$ & $K_{0}(\mathrm{MeV})$ \\
\hline$[5]$ & 9.573 & 1.219 & - & - & 0.556 & 540 \\
{$[14]$} & 8.507 & 0.933 & 1.107 & 41.26 & 0.684 & 285.91 \\
{$[15]$} & 8.897 & 0.947 & 1.073 & 0 & 0.663 & 267.23 \\
Expt. & $\sim 10.145$ & $\sim 1.447$ & - & - & $\sim 0.6$ & $200-300$ \\
\hline
\end{tabular}


The model reproduces well-observed saturation properties of nuclear matter at the saturation density $\rho_{B}=\rho_{0}$. Values of parameters and physical quantities are given in Table 1, based on requiring that

$$
m_{N}=-\tilde{G}_{s} u_{\mathrm{vac}}=939 \mathrm{MeV},
$$

with $u_{\text {vac }}$ satisfying the gap equation (9) taken at vacuum, $T=0$, and $\rho_{B}=0$, and

$$
\mathscr{E}_{\text {bin }}=-m_{N}+\mathscr{E} / \rho_{B} \simeq-15.8 \mathrm{MeV} \text { at } \rho_{B} \simeq 0.17 \mathrm{fm}^{-3} \text { and } T=0 .
$$

The dependence of the binding energy on baryon density is shown in Fig. 1.

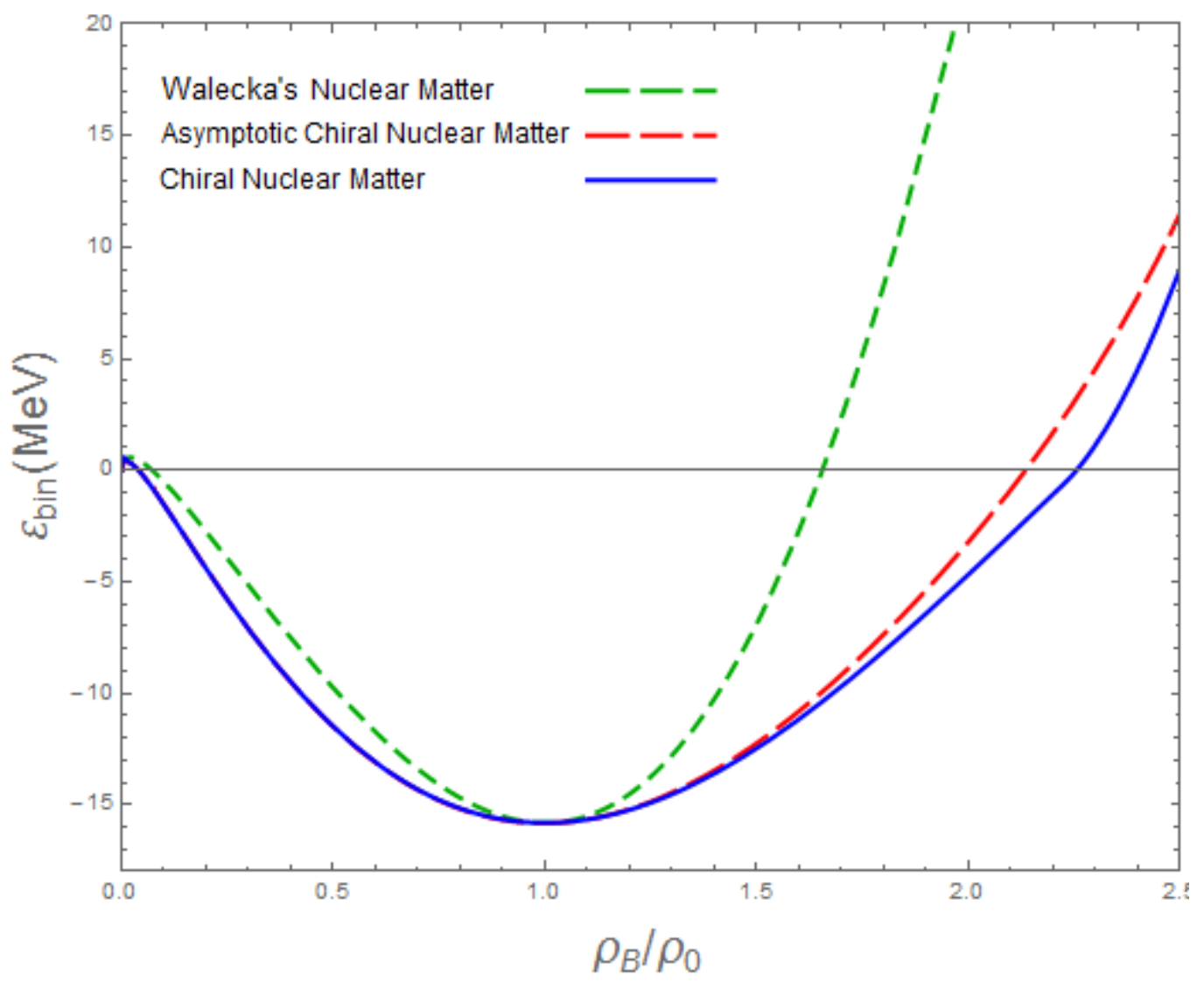

Fig. 1. Nuclear binding energy as a function of baryon density. The green short dashed, red long dashed, and blue solid lines are taken from Refs. [5], [14], and [15], respectively.

The model gives two very interesting results. Firstly, it reveals a first-order liquid-gas phase transition occurring at subsaturated densities, from $\mu_{B} \simeq 923 \mathrm{MeV}$ and $T=0$ and extending to a crossover critical end point (CEP) at $\mu_{B} \simeq 922 \mathrm{MeV}$ and $T \simeq 18 \mathrm{MeV}$. Secondly, the model predicts an exact restoration of chiral symmetry at high baryon densities, $\rho_{B} \gtrsim 2.2 \rho_{0}$ for $0 \lesssim T \lesssim 171$ $\mathrm{MeV}$ and $\mu_{B} \gtrsim 980 \mathrm{MeV}$, and/or at high temperature, $T \gtrsim 171 \mathrm{MeV}$ for $\mu_{B} \lesssim 980 \mathrm{MeV}$ and $\rho_{B} \lesssim 2.2 \rho_{0}$. In the temperature-chemical potential plane a second-order chiral phase transition occurs at $\mu_{B} \simeq 980 \mathrm{MeV}$ and $T=0$, and extends to a tricritical point (CP) at $\mu_{B} \simeq 980 \mathrm{MeV}$ and 
$T \simeq 171 \mathrm{MeV}$, signaling the onset of a first-order phase transition for $T \gtrsim 171 \mathrm{MeV}$. The phase diagram of the two features is displayed in Fig. 2. It displays a clear first-order liquid-gas transition of symmetric nuclear matter at subsaturation and a chiral phase transition of nuclear matter at high baryon density (with the second-order) or at high temperature (with the first-order).

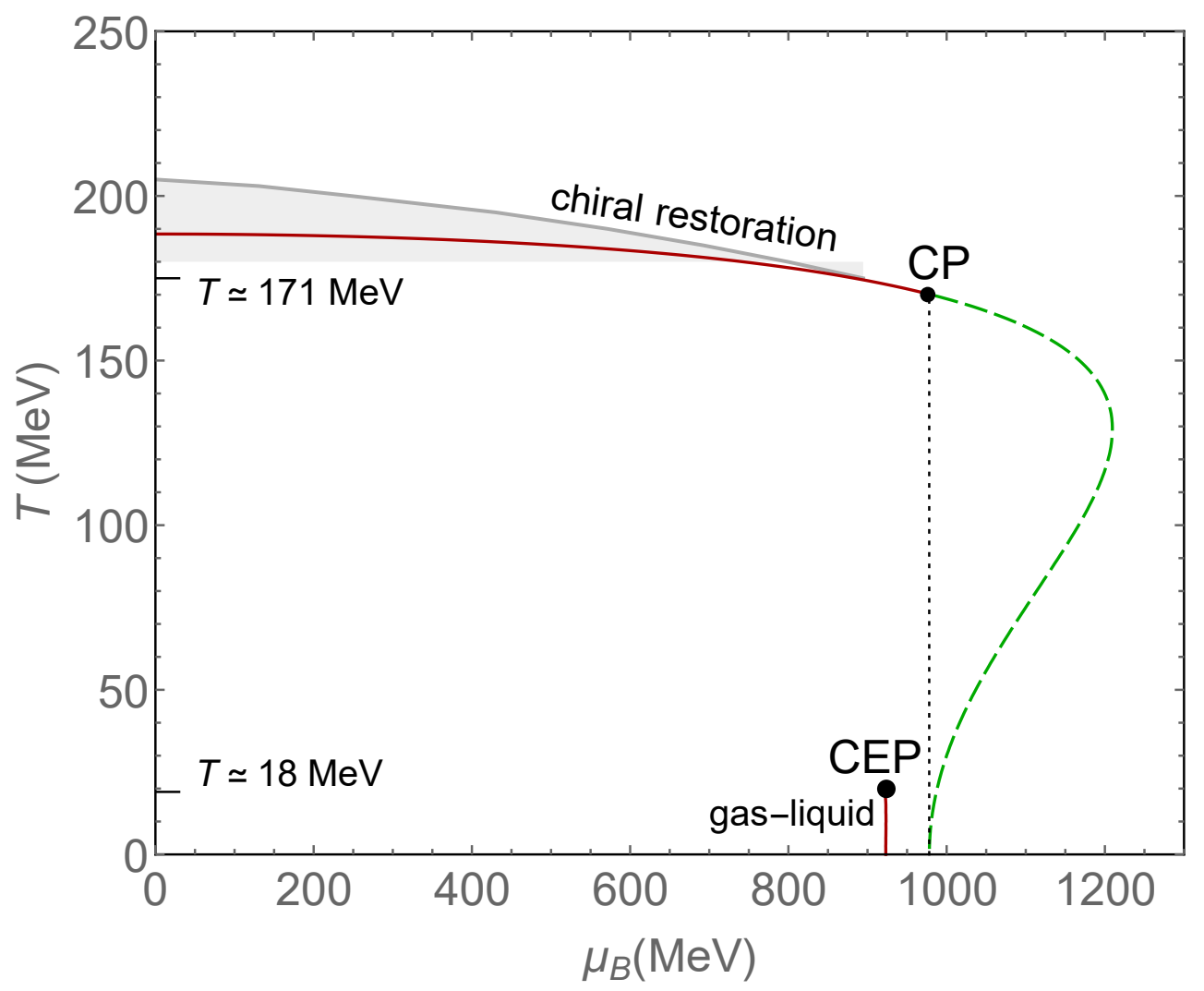

Fig. 2. The phase diagrams of the chiral nuclear matter in the temperature-chemical potential plane. The dashed line denotes a second-order transition. The solid line denotes a first-order phase transition. The critical end point $\left(T \simeq 18 \mathrm{MeV}, \mu_{B} \simeq 922 \mathrm{MeV}\right)$ signs CEP. The tricritical point $\left(T \simeq 171 \mathrm{MeV}, \mu_{B} \simeq 980 \mathrm{MeV}\right.$ ) signs $\mathrm{CP}$, where the line of second-order chiral phase transition meets the line of first-order phase transition. The shadow region is the emergence of hadron-quark mixed phases during the hot chiral phase transition.

\section{THE HADRON-QUARK PHASE TRANSITION}

In this section we discuss the emergence of the inhomogeneous structure associated with the hadron-quark deconfinement transition. For this purpose we need both EOSs of hadron matter and quark-gluon plasma as realistically as possible. As we mentioned in the last section, no one knows how to exactly calculate the hadron-quark phase transition at high density and high temperature regions. The studies by using the effective models of QCD such as the MIT bag model or the NJL model have been actively done instead, we here use the MIT bag model for simplicity. 


\section{III.1. Hadron phase at chiral limit}

We now study the chiral phase transitions at high temperature. Form the phase diagram (Fig. 2) and $\rho_{B}$ dependence of the chiral condensate (Fig. 3), we realize that the chiral phase transition at high temperature is the first-order and above $T \simeq 171 \mathrm{MeV}$. For example at $T=$ $190 \mathrm{MeV}$, the shadow region shows that the chiral condensate is a multivalued function and that it is a mixture state of hot nuclear phase and hot chiral phase.

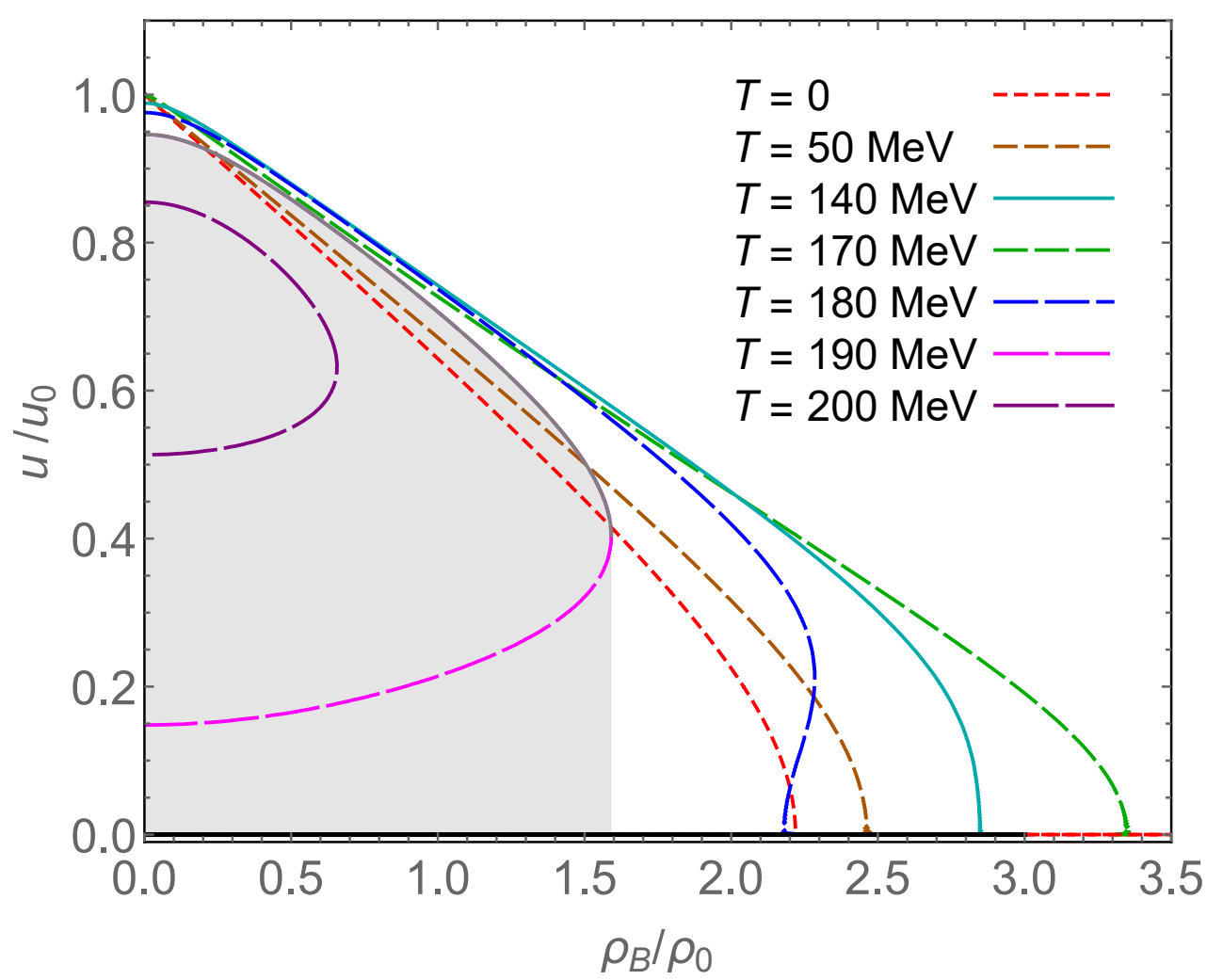

Fig. 3. The $\rho_{B}$ dependence of the chiral condensate at various values of $T$. For example at $T=190 \mathrm{MeV}$, the shadow region shows that there exits a mixture state of hot nuclear phase and hot chiral phase.

Hence, the integral terms in thermodynamic potential, gap equation, baryon density, energy density and EoS can be expanded about chiral limit. Thus, Eqs. (9), (10), (11), and (12) lead

$$
\begin{aligned}
u & \simeq u_{\mathrm{vac}}-\frac{N_{f}}{\pi^{2}} \tilde{G}_{s} u T^{2}\left[\operatorname{Li}_{2}\left(-e^{-\mu^{*} / T}\right)+\operatorname{Li}_{2}\left(-e^{\mu^{*} / T}\right)\right] \\
\rho_{B} & \simeq \frac{N_{f}}{\pi^{2}} T^{3}\left[\operatorname{Li}_{3}\left(-e^{-\mu^{*} / T}\right)-\operatorname{Li}_{3}\left(-e^{\mu^{*} / T}\right)\right]
\end{aligned}
$$




$$
\begin{aligned}
& \mathscr{E}_{\mathrm{HD}} \simeq-\frac{G_{s}}{2} u_{\mathrm{vac}}^{2}+\frac{G_{v}}{2} \rho_{B}^{2}-\frac{6 N_{f} T^{4}}{\pi^{2}}\left[\operatorname{Li}_{4}\left(-e^{-\mu^{*} / T}\right)+\mathrm{Li}_{4}\left(-e^{\mu^{*} / T}\right)\right] \\
& P_{\mathrm{HD}} \simeq-\frac{G_{s}}{2} u_{\mathrm{vac}}^{2}+\frac{G_{v}}{2} \rho_{B}^{2}-\frac{2 N_{f}}{\pi^{2}} T^{4}\left[\operatorname{Li}_{4}\left(-e^{-\mu^{*} / T}\right)+\mathrm{Li}_{4}\left(-e^{\mu^{*} / T}\right)\right] .
\end{aligned}
$$

Here, $\mathrm{Li}_{n}$ is the polylogarithm function of $n$ order $\left(\operatorname{Li}_{n}(z)=\sum_{k=1}^{\infty} z^{k} / k^{n}\right)$.

Figures 2 and 3 show that when $T>171 \mathrm{MeV}$ the chiral condensate can be dropped to zero even at very small values of the baryon density and/or the chemical potential. This is suggested that, when matter is heated sufficiently, hadrons become massless and begin to overlap and then quarks and gluons can freely travel over larger space-time distances. Within this picture, $T \simeq$ $171 \mathrm{MeV}$ is the limiting temperature for the deconfinement phase transition between hadrons and quarks and gluons, that we may call the chiral limit.

At high density and/ or high temperature, the transition beyond the chiral symmetry restoration just after deconfinement is the so-called hadron-quark transition. The system becomes packed densely with nucleons as the density increases to about $2 \rho_{0}$. At higher densities they will even start to overlap. Therefore, one expects that the system in terms of quark and gluon degrees of freedom is more appropriate to described in around densities of a few times nuclear matter ground state density.

Similarly, at high temperature, even at low baryon density, nuclear matter consists not only of nucleons but also of thermally excited hadrons, the temperature affects the momentum scale for scattering events between hadrons. As the temperature is on the order of or higher than $\Lambda_{\mathrm{QCD}}$, scattering between hadrons starts to probe their quark-gluon substructure. Thus, one expects that the system in terms of quarks and gluons is more appropriate to described above a certain temperature.

From these considerations the picture is the following: for very low baryon chemical potential $\mu_{B} \sim 0$, the limiting temperature for HQ phase transition from nuclear matter as a gas of hadrons to plasma of quarks and gluons, corresponding to $P \geq 0$, reads

$$
T_{\min }=\frac{\sqrt{3}}{\pi}\left(\frac{5}{7}\right)^{1 / 4} \Lambda \simeq 202.7 \mathrm{MeV} \text { at } \mu_{B}=0 .
$$

\section{III.2. Quark phase}

We use the standard MIT bag model [16] for massless, non-interacting u, d quarks and gluons for the quark phase. At high density and/ or high temperature we obtain EoS of quarkgluon plasma, i.e.,

$$
P_{\mathrm{QGP}} \simeq \frac{8 \pi^{2} T^{4}}{45}+N_{f}\left(\frac{7 \pi^{2} T^{4}}{60}+\frac{\mu_{q}^{2} T^{2}}{2}+\frac{\mu_{q}^{4}}{4 \pi^{2}}\right)-B .
$$

and other quantities,

$$
\begin{aligned}
& \mathscr{E}_{\mathrm{QGP}} \simeq \frac{8 \pi^{2} T^{4}}{15}+N_{f}\left(\frac{7 \pi^{2} T^{4}}{20}+\frac{3 \mu_{q}^{2} T^{2}}{2}+\frac{3 \mu_{q}^{4}}{4 \pi^{2}}\right)+B, \\
& \rho_{\mathrm{QGP}} \simeq \frac{N_{f}}{3}\left(\mu_{q} T^{2}+\frac{\mu_{q}^{3}}{\pi^{2}}\right) .
\end{aligned}
$$


Here, a baryon consists of three quarks, then $\rho_{B}=\rho_{q} / 3$ and $\mu_{B}=3 \mu_{q}$. In analogy to the blackbody radiation of massless photons, the temperature dependence of the pressure obeys a StefanBoltzmann law. The bag parameter $B$, measured for the energy density of the vacuum, is used to represented the properties of the physical vacuum.

It has been found [17] that with a density-independent bag constant $B$ in the MIT bag model (without color superconductivity), the maximum mass of a neutron star cannot exceed a value of about 1.6 solar masses. As the value of $B$ decreases, the maximum mass increases, but too small values of $B$ are incompatible as a HQ transition density $\rho_{B}>2-3 \rho_{0}$ in nearly symmetric nuclear matter, as demanded by heavy-ion collision.

To overcome these restrictions of the model, a density-dependent bag parameter $B\left(\rho_{B}\right)$ can be introduced, as followed by Ref. [18]. This leads to smaller the value of $B$ at high density (and/or high temperature), while increasing the value of the maximum mass and providing a stiffer QGP EoS, and still fulfilling the condition of no phase transition below $\rho_{B} \approx 2 \rho_{0}$ in symmetric matter. Using a gaussian parametrization for the density dependence, we present the result of $B$ based on the MIT model,

$$
B\left(\rho_{q}\right)=B_{\infty}+\left(B_{0}-B_{\infty}\right) e^{-\beta^{2}\left(\frac{\rho_{q}}{\rho_{0}}\right)^{2}}
$$

with $\beta=0.17$.

The limiting temperature for hadron-quark phase transition, corresponding to $P \geq 0$, reads

$$
T_{\min }=\sqrt{\frac{3}{\pi}}\left(\frac{10}{37}\right)^{1 / 4} B_{0}^{1 / 4} \text { at } \mu_{B}=0 .
$$

Comparing this equation to (19), we get

$$
B_{0}^{1 / 4}=\left(\frac{37}{14}\right)^{1 / 4} \frac{\Lambda}{\sqrt{\pi}} \simeq 287.7 \mathrm{MeV} .
$$

The value of $B_{\infty}$ is fixed at the tricritical point $\left(T \simeq 171 \mathrm{MeV}, \mu_{B} \simeq 980 \mathrm{MeV}\right)$. It gives

$$
B_{\infty}^{1 / 4}=\frac{\Lambda}{\sqrt{\pi}} \simeq 225.7 \mathrm{MeV} .
$$

The bag parameters $B$ found here is inside the range from $B^{1 / 4}=125 \mathrm{MeV}$ to about 300 $\mathrm{MeV}$. This result is consistent with the value analyzed from hadron spectroscopy of the bag model [19].

The thermodynamic quantities in the MIT-Bag model such as pressure and energy density can be calculated as functions of quark chemical potential (or baryon chemical potential) and temperature and the HQ phase transition is inferred via the Gibbs construction of the phase boundary. In the MIT bag model, the HQ transition is of first order, implying that at constant chemical potential the phase boundary is obtained by the requirement that the pressure of the hadronic phase is equal to that in the QGP.

\section{III.3. Phase equilibrium}

The hadronic EoS (18) is matched to the QGP EoS (20) via Gibbs conditions for (mechanical, chemical and thermal) phase equilibrium [20],

$$
P_{\mathrm{HD}}=P_{\mathrm{QGP}}, \quad T_{\mathrm{HD}}=T_{\mathrm{QGP}}, \quad \mu_{\mathrm{HD}}=\mu_{\mathrm{QGP}} .
$$


This leads to a phase boundary curve $T^{*}\left(\mu^{*}\right)$ in the $T-\mu$ plane determined by the equation $P_{\mathrm{HD}}\left(T^{*}, \mu^{*}\right)=P_{\mathrm{OGP}}\left(T^{*}, \mu^{*}\right)$, shown in Fig. 4. Constructed via (27) the phase transition is of first order for $T>171 \mathrm{MeV}$, charactering to a mixed phase of hadron matter and QGP and creating to a latent heat.

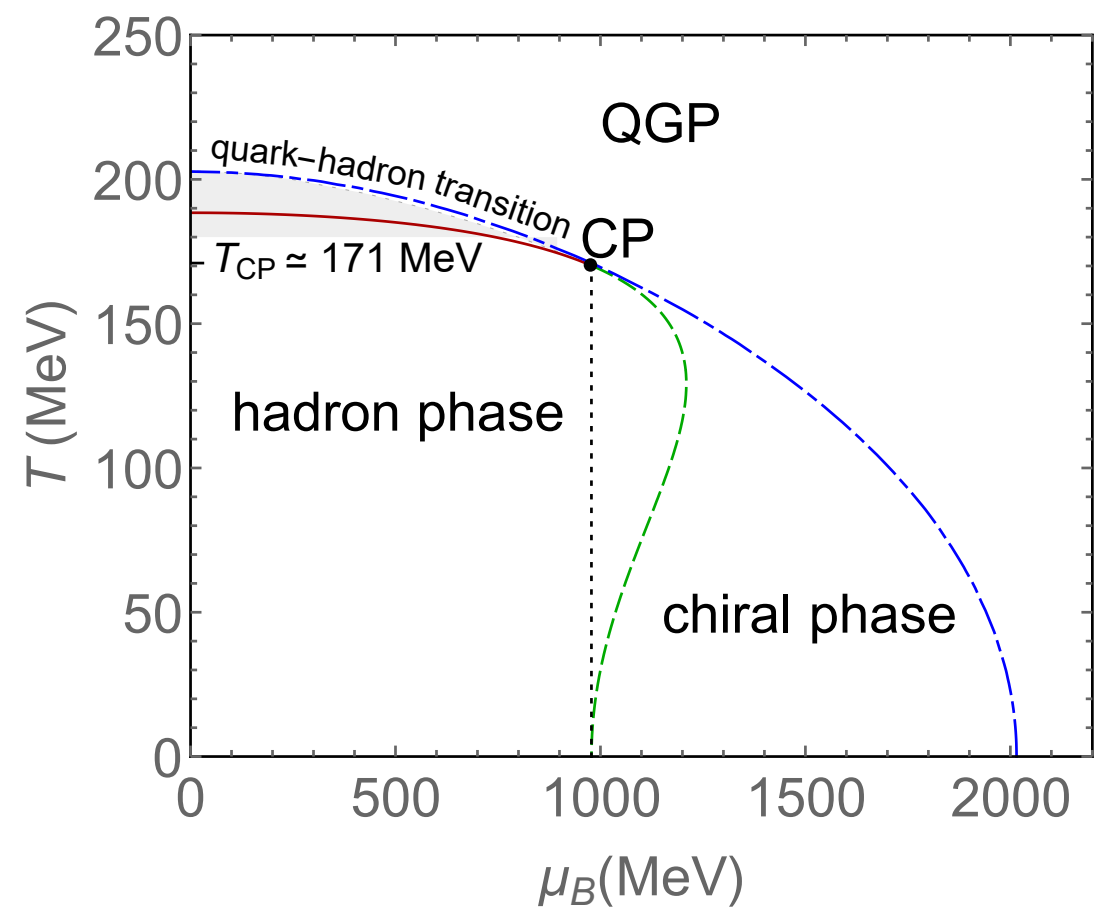

Fig. 4. The hadron-quark phase transitions (blue dot-dashed line) of the hot chiral nuclear matter to quark-gluon plasma in the $\left(T, \mu_{B}\right)$ plane. The shadow region is the emergence of hadron-quark mixed phases during the hot chiral phase transition.

It should be realized from Fig. 4 that there exits a region where the HQ phase transition occurs beyond chiral symmetry restoration in the nuclear phase side, in which the nucleon mass is zero. This is a phase in which nucleonic (hadronic) elementary excitations exist but in chiral symmetry just before the HQ phase transition from the nuclear phase to the quark one. This region which has been proposed recently by McLerran and Pisarski based on large $N_{c}$ arguments [21] the so-called quarkyonic matter as a new state of matter characterized by confinement and chiral symmetry restoration. Thus, the phase beyond the chiral symmetric nuclear phase predicted by our model may corresponds to the quarkyonic phase.

\section{CONCLUSIONS}

The HQ phase transition in the ENJL model included scalar-vector eight-point interaction at very high density and temperature has been investigated following Ref. [15]. As a first attempt to understand the HQ phase transition, we consider the hadron side as chiral nuclear matter and 
the quark side as a QGP without quark-pair correlation. Both phases were matched via Gibbs' phase equilibrium conditions for a first-order phase transition.

There is an interesting region from the phase diagram in Fig. 4 where the HQ phase transition occurs beyond chiral symmetry restoration in the nuclear matter. This region might appear as an exotic phase, not the quark phase, but the nuclear phase, where the chiral symmetry is restored. The so-called quarkyonic phase [21] as a chiral symmetric confined matter may correspond to this phase.

The color superconducting phase which may exist in finite density systems has been ignored in this paper. So, the next challenging tasks may be to consider the other phases of nuclear matter, counting nuclear super-fluidity and QGP, and including the color superconducting state, i.e., quark pairing on the quark phase and nucleon pairing on the nuclear phase. Furthermore, it is widely believed that at high density and/ or high temperature neutron star matter undergoes a phase transition to QGP. Therefore, it is also interesting to investigate the phase transition at high density and/ or temperature between neutron star matter and QGP. This leads to the understanding and the development of the physics of neutron stars.

\section{REFERENCES}

[1] D. J. Gross and F. Wilczek, Phys. Rev. Lett. 30 (1973) 1343; H. D. Politzer, Phys. Rev. Lett. 30 (1973) 1346.

[2] J. C. Collins and M. J. Perry, Phys. Rev. Lett. 34 (1975) 1353

[3] K. Fukushima and T. Hatsuda, Rep. Prog. Phys. 74 (2011) 014001.

[4] T. Schaefer, arXiv:0509068; P. Braun-Munzinger and J. Wambach, Rev. Mod. Phys. 81 (2009) 1031.

[5] B. D. Serot and J. D. Walecka, Advances in Nuclear Physics, Vol.16, eds. J. W. Negele and E. Vogt, Plenum, New York, 1986.

[6] Y. Nambu and G. Jona-Lasinio, Phys. Rev. 122 (1961) 345; ibid. 124 (1961) 246.

[7] S. P. Klevansky, Rev. Mod. Phys. 64 (1992) 649; T. Hatsuda and T. Kunihiro, Phys. Rep. 247 (1994) 221.

[8] M. Buballa, Phys. Rep. 407 (2005) 205.

[9] W. Bentz and A. W. Thomas, Nucl. Phys. A 693 (2001) 138.

[10] Tran Huu Phat, Nguyen Tuan Anh, and Le Viet Hoa, Nucl. Phys. B 772 (2003) c548.

[11] Tran Huu Phat, Nguyen Tuan Anh, Nguyen Van Long and Le Viet Hoa, Phys. Rev. C 76 (2007) 045202.

[12] Tran Huu Phat, Nguyen Tuan Anh and Nguyen Van Long, Phys. Rev. C 77 (2008) 054321.

[13] V. Koch, T. S. Biro, J. Kunz and U. Mosel, Phys. Lett. B 185 (1987) 1.

[14] Tran Huu Phat, Nguyen Tuan Anh, and Dinh Thanh Tam, Phys. Rev. C 84 (2011) 024321.

[15] Nguyen Tuan Anh and Dinh Thanh Tam, Phys. Rev. C 84 (2011) 064326.

[16] A. Chodos, R. L. Jaffe, K. Johnson, C. B. Thorn, and V. F. Weisskopf, Phys. Rev. D 9 (1974) 3471.

[17] G. F. Burgio, M. Baldo, P. K. Sahu, A. B. Santra, and H.-J. Schulze, Phys. Lett. B 526 (2002) 19; G. F. Burgio, M. Baldo, P. K. Sahu, and H.-J. Schulze, Phys. Rev. C 66 (2002) 025802.

[18] O. E. Nicotra, M. Baldo, G. F. Burgio, and H.-J. Schulze, Phys. Rev. D 74 (2006) 123001.

[19] U. Heinz, P. R. Subramanian, H.Stöcker, and W. Greiner, J. Phys. G 12 (1986) 1237; J. Cleymans and E. Suhonen, Z. Phys. C 37 (1987) 51; H. Kouno and F. Takagi, Z. Phys. C 42 (1989) 209; B. M. Waldhauser, D. H. Rischke, J. A. Maruhn, H. Stöcker, and W. Greiner, Z. Phys. C 43 (1989) 411; Q.-R. Zhang and H.-M. Liu, Phys. Rev. C 46 (1992) 2294; Bo-Qiang Ma, Qi-Ren Zhang, D. H. Rischke, W. Greiner, Phys. Lett. B 315 (1993) 29. 
[20] D. H. Rischke, Y. Puersuen, J. A. Maruhn, Nucl. Phys. A 595 (1995) 383; Erratum-ibid. A 596 (1996) 717.

[21] L. McLerran and R. D. Pisarski, Nucl. Phys. A796 (2007) 83; Y. Hidaka, L. McLerran and R. D. Pisarski, Nucl. Phys. A 808 (2008) 117; L. McLerran, K. Redlich and C. Sasaki, Nucl. Phys. A 824 (2009) 86. 